\title{
Dense Multiple Antenna Systems ${ }^{1}$
}

\author{
Nicolae Chiurtu Bixio Rimoldi Emre Telatar \\ Department of Communication Systems \\ École Polytechnique Fédérale de Lausanne \\ CH-1015 Lausanne \\ Switzerland \\ \{Nicolae.Chiurtu,Bixio.Rimoldi, Emre.Telatar\}Qepf1.ch
}

\begin{abstract}
We consider multiple antenna systems in which a large number of antennas occupy a given physical volume. In this regime the assumptions of the standard models of multiple antennas systems become questionable. We show that for such spatially dense multiple antenna systems one should expect the behavior of the capacity to be qualitatively different than what the standard multiple antenna models predict.
\end{abstract}

\section{INTRODUCTION AND MODEL}

In multiple antenna communication systems the signal $Y_{j}$ received at the $j^{\text {th }}$ receiving antenna is related to signals $x_{1}, \ldots, x_{t}$ sent by the transmitting antennas via

$$
Y_{j}=\sum_{i} H_{j i} x_{i}+Z_{j}, \quad j=1, \ldots, r,
$$

where $\left\{H_{j i}: i=1, \ldots, t, j=1, \ldots, r\right\}$ is a collection of random variables independent of the transmitted signals, and $Z_{j}$ is the additive noise, independent of everything else, circularly symmetric and Gaussian.

In most physical scenarios, the gain $H_{j i}$ from the $i^{\text {th }}$ transmitter to the $j^{\text {th }}$ receiver is due to the agglomeration of many small contributions and thus it is reasonable to assume that the collection $\left\{H_{j i}\right\}$ is jointly Gaussian, and circularly symmetric. Furthermore, it is also common to assume that the $\left\{H_{j i}\right\}$ form an independent collection.

While this last assumption can be justified in cases for which the transmitting antennas are separated from each other by some multiple (e.g., 1/4) of the wavelength and likewise for the receiving antennas, when we wish to talk about a large number of antennas packed into a fixed volume this assumption becomes questionable. Furthermore, if one fixes the variances of $H_{j i}$ and holds the total transmitted power constant, the total signal power received by the receiving antennas scales with $r$. If the receiving antennas are assumed to occupy a given volume, to assume that one can increase the total received power by placing more receiving antennas into this volume sounds very dubious.

The concerns raised above leads us to consider a multiple antenna system with $r$ receiving and $t$ transmitting antennas where

$$
Y=r^{-1 / 2} H x+Z .
$$

\footnotetext{
${ }^{1}$ This work was supported in part Nokia/diAx/SBC.
}

The scaling of the information carrying component $H x$ of the received signal by $r^{-1 / 2}$ ensures that the total received power remains bounded even when we increase $r$. We will assume that the entries of the channel gain matrix $H$ are jointly circularly symmetric and Gaussian, and have a correlation structure given by

$$
E\left[H_{j i} H_{l k}^{*}\right]=C_{k i} D_{j l} \text {. }
$$

One justification for the above form is the following: imagine that the signals sent from the transmitted antennas first travel to a 'cloud' of scatterers, and then from this cloud they travel to the receiving antennas. Assume that once in the cloud, the signals forget wherefrom they came. This translates to the assumption that $H_{j i}=A_{i} B_{j}$ where $A_{i}$ is the gain from the transmitter $i$ to the cloud, $B_{j}$ the gain from the cloud to the receiver $j$ and that the ' $A$ ' gains and ' $B$ ' gains are independent. However, it is likely that when antennas $i$ and $k$ are close to each other $A_{i}$ and $A_{k}$ will be correlated, and similarly for $B_{j}$ and $B_{l}$. If we let these correlations be $C_{k i}$ and $D_{j l}$ we obtain (2). It now also becomes clear that we should assume that $C$ and $D$ are non-negative definite matrices. Note that the same correlation structure is assumed in [1].

For the purposes of this abstract, assume (quite optimistically) that the receiver is aware of the realization of $H$, but that the transmitter only knows the statistics of $H$, namely, $C$ and $D$.

\section{RESULTS}

To calibrate our intuition, consider first the case where $C=I_{t}, D=I_{r}$, in other words, when the $H_{j i}$ are uncorrelated. Notice that this takes into account the power penalty at the receiver, but ignores the possible correlations between the path gains. From [2] we know that in this case the capacity is given by

$$
\mathrm{C}=E\left[\log \operatorname{det}\left(I+\frac{P}{r t} H H^{\dagger}\right)\right] .
$$

If we let $\mu_{1}, \ldots, \mu_{n}$ denote the eigenvalues of $H H^{\dagger}$, we can rewrite the above as

$$
\mathrm{C}=\sum_{i} E\left[\log \left(1+P \mu_{i} / r t\right)\right]
$$

Using $\log (1+z) \leq z$, and noticing that $\sum_{i} \mu_{i}=\operatorname{tr}\left(H H^{\dagger}\right)$, we obtain

$$
\mathrm{C} \leq \frac{P}{r t} \operatorname{tr}\left(E\left[H H^{\dagger}\right]\right)=P .
$$


On the other hand, for $z \geq 0, \log (1+z) \geq z-z^{2} / 2$. Since $\sum_{i} \mu_{i}^{2}=\operatorname{tr}\left(H H^{\dagger} H H^{\dagger}\right)$, we obtain

$$
\mathrm{C} \geq P-\frac{P^{2}}{2(r t)^{2}} \operatorname{tr}\left(E\left[H H^{\dagger} H H^{\dagger}\right]\right)=P-\frac{P^{2}(r+t)}{2 r t} .
$$

We thus see that for a fixed $P$, as $r$ and $t$ increases

$$
\lim _{r, t \rightarrow \infty} \mathrm{C}=P \text {. }
$$

Contrast this expression with the usual multiple antenna case where the capacity increases linearly with increasing number of antennas.

Let us now bring in the path gain correlations due to the proximity of the receiving antennas to each other. Consider a fixed volume of space into which we pack more and more receiving antennas. In this case, we can label the antennas such that $(j-1) / r$ indicates the physical location of antenna $j$ when $r$ receiving antennas are present. It now makes sense to assume that the correlation between the $j^{\text {th }}$ and $l^{\text {th }}$ receiving antennas is a function of their physical positions only, thus, $D_{j l}$ is a function of $(j-1) / r$ and $(l-1) / r$, i.e., $D_{j l}=d((j-1) / r,(l-1) / r)$. Since $d:[0,1]^{2} \rightarrow \mathbb{C}$ models a covariance function, it is Hermitian and positive definite, i.e., $d(\alpha, \beta)=d(\beta, \alpha)^{*}$, $\iint g(\alpha)^{*} d(\alpha, \beta) g(\beta) d \alpha d \beta \geq 0$ for any $g$. In addition, let us assume that $d$ is continuous and that

$$
\iint|d(\alpha, \beta)|^{2} d \alpha d \beta<\infty
$$

We will say that such a system is spatially dense at the receiver. For the time being let the transmitting antennas be 'sparse', i.e., suppose that $C=I_{t}$.

With these assumptions, one can show that the eigenvalues of the matrix $D / r$ for large $r$ approach the (point) spectrum of the operator $d$ : Notice first that the operator $d$ only has a point spectrum (see, e.g. [3]) and that the eigenvalues of $D / r$ are exactly those of the operator $d_{r}:[0,1]^{2} \rightarrow \mathbb{C}$, where

$$
d_{r}(\alpha, \beta)=d(\lfloor r \alpha\rfloor / r,\lfloor r \beta\rfloor / r) .
$$

Second, note that for any $L_{2}[0,1]$ function $g$,

$$
\iint g(\alpha)^{*} d_{r}(\alpha, \beta) g(\beta) d \alpha d \beta \rightarrow \iint g(\alpha)^{*} d(\alpha, \beta) g(\beta) d \alpha d \beta
$$

with increasing $r$. Combining this with the extremal representation of the eigenvalues of Hermitian operators and matrices we see that the eigenvalues of $D / r$ approach the spectrum of $d$.

It is now easy to see that the capacity of this system in the limit of large $r$ and $t$ approaches a finite limit.

If one assumes that the transmitting antennas are also spatially dense, then a more involved argument leads one to conclude that the effect of large $r$ and $t$ on the capacity is to scale the power by a factor $t$. In other words, if $\mathrm{C}(r, t, P)$ denotes the capacity for a given number of receiving and transmitting antennas and available power $P$, $\mathrm{C}(r, t, P)$ behaves like $F(t P)$ for large $r$ and $t$ for a suitable function $F$. This seems to be due to the feasibility in the model of beam-forming at the transmitter when there is a large amount of correlation between the gains from transmitting antennas to the scattering medium. One wonders how physically feasible beam-forming is; perhaps an additional constraint on the covariance matrix of the transmitted signals $x$ is necessary to more accurately reflect the physical constraints.

\section{CONCLUSION}

Spatially dense multiple antenna systems exhibit qualitatively different behavior from their sparse counterparts in the limit of large number of antennas. This paper attempts to point out the gross differences between such systems by focusing on the case of complete channel side information at the receiver. As of this writing, we have partial results that relate to the case of no channel side information, we will report on our progress shortly.

\section{REFERENCES}

[1] D.-S. Shiu, G. J. Foschini, M. J. Gans and J. M. Kahn, "Fading correlation and its effect on the capacity of multielement antenna systems," IEEE Transactions on Communications, v. 48, no. 3, pp. 502-513, March 2000.

[2] I. E. Telatar, "Capacity of multi-antenna Gaussian channels," AT\&T Bell Laboratories Technical Report, BL0112170-95061507TM, June 1995.

[3] F. Riesz and B. Sz.-Nagy, Functional Analysis, New York, Dover Publications, 1990.

[4] A. M. Sengupta and P. P. Mitra, "Capacity of multivariate channels with multiplicative noise: I. Random matrix techniques and large- $N$ expansions for full transfer matrices," Lucent Technologies Bell Laboratories Technical Report, May 1999. 